\title{
Motivaciones de uso de las redes sociales para el desarrollo del capital social de las mujeres de entorno rural
}

\section{Motives for using social networking sites in the development of rural women's social capital}

\section{Luisa Vega Caro}

Profesora en el Departamento de Métodos e Investigación Educativa (Universidad de Sevilla)

Alba Vico Bosch

Doctoranda (Universidad de Sevilla)

\section{Ángeles Rebollo Catalán}

Profesora en el Departamento de Métodos e Investigación Educativa (Universidad de Sevilla)

Fecha de recepción: 30 de marzo de 2015

Fecha de revisión: 20 de junio de 2015

Para citar este artículo: Vega Caro, L., Vico Bosch, A. y Rebollo Catalán, A. (2015): Motivaciones de uso de las redes sociales para el desarrollo del capital social de las mujeres de entorno rural, Icono 14, volumen (13), pp. 142-162. doi: 10.7195/ri14.v13i2.839 


\section{Resumen}

Estudios previos han mostrado los riesgos de exclusión digital de comunidades rurales y personas mayores y cómo las motivaciones de uso influyen en las prácticas y hábitos de uso de las tecnologías. Con este trabajo queremos analizar cómo las motivaciones, que impulsan a las mujeres adultas de entorno rural a usar las redes sociales, favorecen el desarrollo de su capital social potenciando diferentes formas de participación en la cultura digital. Partiendo de una investigación más amplia sobre 478 mujeres andaluzas de zonas rurales de 18 a 65 años, este trabajo presenta un estudio cualitativo en profundidad de 13 de ellas mediante entrevistas autobiográficas sobre su experiencia en las redes. Los resultados muestran que el uso de las redes sociales descubre oportunidades de participación social vinculadas a diversas inquietudes, revelando las redes sociales como un espacio significativo para promover su participación en la vida cultural y social de sus comunidades.

Palabras clave: Motivos de uso - Capital social - Redes sociales - Estudios de las mujeres - Mujer rural

\section{Abstract}

Previous studies have shown the risk of digital exclusion of rural communities and older people, and how their motives of use influence their practices and use habits of technologies. With this work we analyse how the motives of use that drive adult women in rural areas to use social media, promote the development of their social capital by means of strengthening different forms of participation in digital culture. Starting from a broader investigation on 478 Andalusian rural women 18 to 65 years, this paper presents a qualitative study in depth with 13 of them by autobiographical interviews about their experience in networks. The results show that the use of social networking sites discover them opportunities for social participation linked to various interests, revealing social networks as significant environment to promote their participation in cultural and social life of their communities.

Key Words: Motives of use - Social capital - Social networks - Women's studies Rural women

ICONO14 | Año 2015 Volumen 13 Nº 2 | ISSN: 1697-8293 | DOI: ri14.v13i2.839 


\section{Introducción}

La mayor parte de las investigaciones previas sobre el uso de las tecnologías por parte de mujeres adultas han mostrado que las motivaciones que las impulsan son de carácter relacional (Arteaga, Cortijo \& Javed, 2014; Dias, 2012; Hew \& Cheung, 2012; Jiménez-Cortés, Rebollo-Catalán, García-Pérez \& Buzón-García, 2015; Lin \& Lu, 2011; Rebollo-Catalán \& Vico-Bosch, 2014; Yang \& Brown, 2013) así como por su aplicabilidad o utilidad directa para su vida cotidiana (Huber \& Watson, 2014; Vergés, Haché \& Cruells, 2011).

Paralelamente las investigaciones sociológicas que se han centrado en el análisis de las redes sociales virtuales han documentado su papel en la promoción y desarrollo del capital social de grupos, comunidades y personas (Falk \& Kilpatrick, 2000; Kilpartrick, Field \& Falk, 2003; Sánchez-Franco, Buitrago-Esquinas \& Hernández-Mogollón, 2012).

No obstante, pocos estudios han profundizado en la relación entre motivos y capital social, analizando de qué forma las motivaciones de uso impulsan o promueven el capital social de las personas y su forma de expresarse o comunicarse a través de estos nuevos medios (García, López de Ayala \& Catalina, 2013; Greenhow \& Burton, 2011; Pfeil, Arjan \& Zaphiris, 2009; Tosun, 2012).

Algunos informes advierten de las especiales dificultades de las comunidades rurales (LaRose, Gregg, Strover, Straubhaar \& Carpenter, 2007; Novo-Corti, VarelaCandamio \& García-Álvarez, 2014) y de las personas mayores (Braun, 2013; Dias, 2012) en el acceso y uso de las tecnologías, señalando los múltiples factores que pueden incidir en su exclusión digital. Los últimos informes en España (Plan de Inclusión Digital y Empleabilidad, 2013) indican que sólo el 62\% de la población accede de forma regular a Internet, lo que supone que casi un $40 \%$ de los ciudadanos no accede a las TIC, de los cuales el $22,7 \%$ no ha usado nunca Internet suponiendo el mayor porcentaje en este grupo las personas entre 55 y 64 años, lo que indica la existencia de una brecha generacional. Algunas investigaciones (DiMaggio, Hargittai, Celeste \& Shafer, 2004; Hilbert, 2011; van Dijk, 2005) también apuntan la existencia de esta brecha que refleja diferencias en el uso de Internet con respecto 
a la edad, siendo una de las más duras, porque supone formas de exclusión a distinto nivel (Vázquez-Gómez, 2003).

Rebollo-Catalán y Vico-Bosch (2014) consideran que los entornos virtuales suponen un espacio que favorecen la participación colaborativa de las personas usuarias de esta herramienta. Esto ha creado nuevas posibilidades para la educación y el aprendizaje, pero también para la exclusión social (DiMaggio et al., 2004; Livingstone \& Helsper, 2007), siendo un entorno clave para el ejercicio de la ciudadanía que depende de factores sociales como la edad, la clase social, el género, el nivel educativo o la ubicación geográfica.

Por ello, las políticas sociales de la última década en Europa han tenido como línea estratégica la inclusión y ciudadanía digital de las mujeres, destacando la Agenda Digital Europea y el Plan de Inclusión Digital y Empleabilidad en los que plantean la alfabetización en competencias digitales para el fomento de la participación de las mujeres en entornos digitales.

\section{Antecedentes}

\subsection{Motivos de uso de las redes sociales en mujeres de zonas rurales}

Las redes sociales virtuales han cambiado la presencia y participación de las mujeres en entornos virtuales, suponiendo un cambio en el panorama de las relaciones interpersonales y en la comunicación entre personas y grupos y, sobre todo, en mujeres de zonas rurales y de edad avanzada. El acceso a Internet parece mejorar las conexiones con el mundo exterior y puede ayudar a evitar o reducir los sentimientos de aislamiento social (Bimber, 2000; Bradley \& Poppen, 2003; Carpenter \& Buday, 2007; Rebollo-Catalán \& Vico-Bosch, 2014). En esta misma línea, otros estudios han señalado el valor de las redes sociales virtuales como un medio para traspasar los límites físico-geográficos de las comunidades permitiendo, por un lado, reforzar comunidades locales y ayudando a mantenerlas unidas y, por otro, desarrollar vínculos con personas con intereses y valores comunes que se encuentran lejanas geográficamente (Castells, 2001; Kollock \& Smith, 2003).

ICONO14 | Año 2015 Volumen 13 Nº 2 | ISSN: 1697-8293 | DOI: ri14.v13i2.839 
Aires, Dias, Azevedo, Rebollo-Catalán y García-Pérez (2014) ponen de manifiesto los cambios que se han producido en los roles tradicionales de los usuarios de las redes, pasando de ser consumidores pasivos a agentes que transforman y producen conocimiento. En relación a ello, varios estudios (Arteaga, Cortijo \& Javed, 2014; Hew \& Cheung, 2012; Yang \& Brown, 2013; Rebollo-Catalán \& Vico-Bosch, 2014) manifiestan que las mujeres acceden a ellas en función de una serie de motivos, entre los que destacan motivos relacionales, de información y entretenimiento. Sin embargo, no podemos decir que el único motivo de uso de las redes sociales por parte de mujeres de zonas rurales sea entablar relaciones con otras personas. Vergés et al. (2011) manifiestan que en cuanto a las motivaciones de acceso sus experiencias muestran una gran variabilidad de motivos que a menudo se superponen. Esto coincide con otros estudios internacionales (Hargittai, 2010; Jiménez-Cortés et al., 2015; Livingstone \& Helsper, 2007) que demuestran la estrecha relación entre una gran diversidad de motivos de uso de las tecnologías y la inclusión digital. No obstante la literatura previa coincide en señalar como una de las motivaciones principales de las mujeres el deseo de explorar, la curiosidad o la atracción, al mismo nivel que otras con finalidades más utilitaristas. Huber y Watson (2014) encuentran que son las personas mayores las que más usan las tecnologías por su utilidad o practicidad en la vida cotidiana. En su estudio señalan la importancia de desarrollar estrategias que ayuden a este colectivo a adaptarse a las tecnologías y a los cambios que puedan producirse en ella, a través de familiares, amigos, ayuda en línea, personal técnico,... Esto concuerda con el estudio de Arteaga et al. (2014), pues no es únicamente la utilidad percibida la que se muestra como factor importante para predecir la adopción de las tecnologías por parte de estas personas, sino también la influencia social y los contextos de uso lo que incide en las actitudes y el cambio de prácticas digitales.

\subsection{El desarrollo del capital social en las redes sociales virtuales}

El concepto de capital social está muy vinculado al uso de las redes sociales virtuales y su influencia en las nuevas alfabetizaciones que implican una ciudadanía digital. Falk y Kilpatrick (2000) y Kilpatrick, Field y Falk (2003) lo definen como la acumulación de recursos de identidad de una comunidad con intereses comunes, que se produce mediante la interacción y colaboración de sus participantes. Estos

DOI: ri14.v13i2.839| ISSN: 1697-8293 | Año 2015 Volumen 13 N² | ICONO14 
recursos son de utilidad para el proceso de desarrollo de la comunidad y conlleva la pertenencia a redes sociales, las normas que rigen las interacciones de sus integrantes, así como la confianza del grupo y la construcción de conocimientos. Esto repercute no sólo en beneficio de la comunidad sino también en la persona, lo cual dependerá de la capacidad que cada una tenga de sacarle provecho en beneficio propio.

Estudios recientes basados en el capital social (Baker, Bricout, Moon, Coughlan \& Pater, 2013; Greenhow \& Burton, 2011; Muscanell \& Guadagno, 2012) han demostrado que entre los beneficios de las tecnologías no sólo están las oportunidades que ofrecen de fortalecer lazos con la propia comunidad y empoderarse dentro de ella, sino de abrir puentes hacia otros contextos, personas y recursos, con especial mención a la mejora profesional y laboral que supone el acceso a información útil sobre oportunidades formativas y profesionales que de otro modo no están disponibles. El ejercicio de una ciudadanía efectiva y plena requiere de competencias digitales para participar en la sociedad de la información, ya que las nuevas tecnologías de la comunicación han cambiado la naturaleza y alcance de la ciudadanía, siendo necesarias nuevas alfabetizaciones (Area \& Pessoa, 2012; Meyers, Erickson \& Small, 2013; Simsek \& Simsek, 2013).

En un estudio sobre la red social Tuenti (Sánchez-Franco et al., 2012) evidencian que la red social virtual es un entorno que promueve la interdependencia entre las personas y su capital social, fomentando su participación activa junto al compromiso comunitario. En particular, este estudio muestra que el compromiso comunitario se ve estimulado por la implicación de la persona en la comunidad y por los intercambios relacionales y su fortaleza, descubriendo que la red social virtual es una fuente de sentimientos de identidad social e individual, pertenencia e implicación comunitaria.

Las políticas sociales en la última década en España y Andalucía han tenido como línea estratégica la inclusión y ciudadanía digital de las mujeres con atención a determinados grupos (edades avanzadas, zonas rurales, etc.). Desde esta perspectiva diversos estudios (Greenhow \& Gleason, 2012; Greenhow \& Robelia, 2009; Hall, 2009) han puesto de relieve la necesidad de desarrollar estrategias

ICONO14 | Año 2015 Volumen 13 N² 2 | ISSN: 1697-8293 | DOI: ri14.v13i2.839 
educativas aprovechando estos nuevos entornos con fines formativos para ampliar el capital social de los ciudadanos.

Por todo ello, con este trabajo nos proponemos los siguientes objetivos:

- Conocer las motivaciones que impulsan a las mujeres adultas de entorno rural a usar las redes sociales virtuales.

- Comprender cómo las motivaciones de uso de las redes por parte de estas mujeres favorecen el desarrollo de su capital social.

\section{Material y métodos}

Este trabajo se enmarca dentro de un estudio más amplio sobre el uso que las mujeres de zonas rurales hacen de las redes sociales virtuales, en el que participan 478 mujeres de edades comprendidas entre 18 y 65 años, las cuales fueron reclutadas a través de su participación en programas y actividades de formación sobre TIC en sus comunidades. Para profundizar en las respuestas obtenidas en una encuesta sobre motivos y hábitos de uso, realizamos un conjunto de estudios de casos con mujeres que mostraron una gran heterogeneidad y versatilidad en las formas de uso de las redes sociales a través de las respuestas en esta encuesta. Fueron seleccionadas 13 mujeres con una experiencia de uso medio mayor o igual a 4 años, mediante un muestreo teórico de casos discrepantes, buscando la máxima variabilidad en perfiles sociodemográficos (edad, situación familiar, nivel educativo y actividad profesional) y experiencia de uso de las redes sociales.

Hemos optado por una metodología cualitativa, aplicando el método autobiográfico con un diseño de relatos paralelos para obtener el testimonio subjetivo que las participantes hacen de su experiencia con las redes sociales. De este modo, es posible aproximarse al recorrido vital de estas personas globalmente, atendiendo a los aspectos personales, sociales y culturales del contexto que les influyen directamente en sus motivos de uso de las redes sociales. Se trata de un método especialmente consolidado en el estudio de trayectorias profesionales (Wagner, Birbaumer y Tolar, 2003), 
así como en el análisis de procesos de exclusión cuando éstos se plantean desde una perspectiva de género (Sancho, 2009). En nuestro caso, y de acuerdo con los objetivos de la investigación, con el desarrollo de las entrevistas en profundidad se ha recogido el testimonio subjetivo de la experiencia específica de relación con las redes sociales de cada una de las mujeres. La entrevista tiene la capacidad de, por un lado, permitirnos recoger gran cantidad de información de manera directa y cercana (Mayorga, 2004), y por otro, establecer cierta relación entre la propia evolución de la persona y las circunstancias personales y sociales que rodean a dicha evolución. El guion de la entrevista fue elaborado a partir de la revisión bibliográfica llevada a cabo. En dicho guion se tuvieron en cuenta las distintas temáticas y aspectos que nos permitieran indagar en las formas de uso de las redes sociales a nivel personal y los motivos por los que la utilizan, así como los beneficios e impactos percibidos en sus vidas.

Las entrevistas fueron grabadas y transcritas, y el análisis del contenido de la información recogida en las mismas se ha desarrollado en cinco etapas, inspirándonos en la propuesta de Strauss y Corbin (1998), según la cual estas etapas avanzan de forma espiral, retomándose constantemente hasta la saturación, es decir hasta la no aparición de nueva información:

1. Segmentación: obtuvimos segmentos informativos de las entrevistas más precisos y claros.

2. Codificación inicial: definimos las principales temáticas que hacían referencia a los diferentes usos que las mujeres hacían de las redes sociales que se encontraban enunciados en los relatos de las entrevistadas.

3. Clasificación y relación entre los códigos iniciales: se relacionaron los códigos teniendo en cuenta el punto en que se encontraban dentro de la entrevista autobiográfica.

4. Codificación focalizada: consistió en la organización y reducción de los códigos en base a la creciente organización en categorías que tomaban los datos, de modo que, así, convertimos dichos códigos en códigos fundamentados, aptos para proseguir la codificación de los datos de forma selectiva.

ICONO14 | Año 2015 Volumen 13 Nº 2 | ISSN: 1697-8293 | DOI: ri14.v13i2.839 
5. Contextualización: identificamos las relaciones entre los códigos fundamentados para definir la línea argumentativa de cada uno de ellos. Concretamos y consensuamos qué los distinguía entre ellos y los contextualizamos en el marco de los objetivos del estudio.

En resumen, el análisis del contenido de las entrevistas se llevó a cabo del tal forma que a partir de sucesivas lecturas de las entrevistas, de su codificación e interpretación de los datos hemos tratado de descubrir patrones sistemáticos o categorías que nos permitan describir, interpretar y comprender cómo las redes sociales promueven el capital social de las mujeres de zonas rurales.

Como consecuencia de este proceso, las categorías de análisis que hemos usado han sido:

a. Motivos de uso: hace referencia a aquellos aspectos que captan la atención de las mujeres y hacen que estas se sientan atraídas y motivadas por usar las redes sociales. Tomando como referencia la clasificación que realizan Papacharissi \& Mendelson (2011), en este estudio hemos identificados de tipos de motivaciones orientadas a:

a.1. Relaciones sociales: donde se incluyen aquellos motivos relacionados con la comunicación, el hacer o mantener amistades, así como, el retomar contactos, es decir, todas aquellas acciones que fomenten la participación social

a.2. Ocio y tiempo libre: se incluyen todas aquellas actividades que les sirvan para entretenerse, disfrutar y relajarse.

a.3. Desarrollo profesional: formado por aquellos motivos de uso vinculado al ámbito laboral.

a.4. Gestión familiar: este tipo de motivación está enfocada al cuidado y la gestión familiar.

b. Capital social: definido como el conjunto de recursos compartidos por una red de relaciones duradera, de conocimiento y reconocimiento mutuo, en la que cada persona como miembro del grupo tiene algo que aportar y recibir del mismo (Bourdieu, 1985 en Straubhaar, Spence, Tufekci \& Lentz (Eds.), 2012). Esta categoría está formada por:

DOI: ri14.v13i2.839| ISSN: 1697-8293 | Año 2015 Volumen 13 N² 2 | ICONO14 
b.1. Fortalecimiento del círculo cercano: alude a las personas con las que se mantiene una estrecha relación con el fin de fortalecer los lazos con su comunidad y con la que comparten intereses y valores.

b.2. Ampliación del círculo: se trata de vínculos estratégicos orientados a mantener relaciones duraderas con otras redes o grupos a lo largo de un tiempo (compañeros de escuela, asociaciones,...).

\section{Resultados}

Los resultados muestran que la motivación principal de uso de las redes sociales por parte de las mujeres se vincula al mantenimiento de relaciones familiares y de amistad, lo que las impulsa en un inicio a usarlas. En reiteradas ocasiones, estas mujeres expresan su necesidad de entrar en las redes para continuar fortaleciendo sus vínculos con familiares y amigos. El siguiente extracto es un claro ejemplo de esta dinámica:

(A): Yo soy de un pueblo de Huelva y Facebook me permite vivir todo lo de allí a través de mi gente. El otro día era la Romería y puse que estaba planchando el traje y ya todos sabían que iba yo para allá. Me gusta mucho escribir, escribo poesía a la Virgen, a una amiga mía muy querida que murió y puedo compartirlo. Lo que más me gusta es que me puedo relacionar con quien quiero.

En este extracto, Ana expresa el valor que las redes sociales tienen en su vida para poder continuar participando en las prácticas culturales de su pueblo de origen a través de la interacción con sus familiares y amigos a pesar de la distancia geográfica.

Los resultados también muestran que este impulso inicial de uso de las redes sociales se produce por el contacto e interacción con personas jóvenes, siendo las redes intergeneracionales las que más apoyo inicial proporcionan a estas mujeres para usarlas. Los extractos siguientes ilustran muy claramente el impacto positivo que supone el mantenimiento de las relaciones familiares con personas más jóvenes como hijas/os, sobrinas/os, primas/os,...

(D): Sí, sí el Facebook porque como ahí tengo mmm prácticamente, ¿no? Sí, 
prácticamente ahí tengo todos los hijos pues por ahí me comunico con ellos, porque mis hijos empezaron a animarme, que ellos estaban ahí, que era una cosa bastante curiosa, y mis nietos también.

(T): Entré en Facebook por el tema del diálogo del día a día "pues yo estoy en tal sitio", "pues, mira prima que te voy a mandar a través del Tuenti esta foto a mi hija", ¿no?, por ejemplo. Y dice "mira, pues la tita también está en el Tuenti". Y digo "pues yo, ¿por qué no voy a estar yo también en el Tuenti?..."

A pesar de que la entrada en las redes sociales por parte de estas mujeres adultas de zonas rurales viene motivada inicialmente por su necesidad de mantenerse en contacto con la familia y amigos íntimos, los resultados muestran de forma persistente el sentido de realización personal que supone progresivamente para ellas a través de las posibilidades que les ofrecen de desarrollar hobbies e intereses. En múltiples pasajes expresan la sorpresa que ha supuesto para ellas poder dar rienda suelta a sus inquietudes en distintos temas que les gustan y en los que se sienten realizadas (cocina, pintura, moda, viajes, etc.). Esto puede verse reflejado en los siguientes extractos:

(I): Comencé a usarla porque me dijo una compañera “imira que hay un grupo de la gente del trabajo antiguo...!" que no sabía yo ni abrirme una cuenta en Facebook, pero iba preguntando a las niñas “bueno, y ahora ¿qué hago?” Y entonces, a partir de eso ya me pongo yo sola y hablo con una, con otra,... Al principio no entiendes, pero una vez que eso, ya le he cogido yo el rollo, y ya me meto en más cosas, busco muchas cosas de recetas o cosas que yo quiera, ¿sabes?

(IR): Yo lo que me quedo es eso, que puedo echar un rato con mis amigos, con algún familiar y que yo muchas veces pues me meto por ahí y veo muchas cosas. Por ejemplo a mí me gustan los complementos, muchas veces pues a lo mejor alguien me pasa información, o yo se la paso "mira que esto se hace así, mejor así". Si me encuentro a lo mejor a alguien y digo ¡uy! Pues mira, esta mujer hace lo mismo que yo, voy a mirar lo que tiene. Sí, a lo mejor le he dado a tener una amistad y me devuelven muchas amistades la gente.

DOI: ri14.v13i2.839| ISSN: 1697-8293 | Año 2015 Volumen 13 N² 2 | ICONO14 
Este contacto con otras personas cercanas a través de las redes rápidamente les descubre nuevas posibilidades y comienzan a usarlas con otros fines despertando su curiosidad por experimentar nuevas facetas. De esta forma, el siguiente extracto muestra un uso vinculado al ámbito laboral:

(A): Yo lo he usado sobre todo para dar a conocer lo que hago. Yo soy presentadora de Tupperware y Starhome, y me hice mi propio Facebook para esto y me llama mucha gente. Pongo mis promociones, ofertas y fotografías de los productos, y me llama mucha gente. Ah y ahora estoy haciendo pendientes de croché preciosos y también ropita para niños de punto o croché. Luego también lo uso por otros temas como ya he dicho y me sirve para que me conozcan y llegar a más personas.

En este relato, Ana revela cómo usos laborales de las redes despiertan un espíritu emprendedor, desarrollando nuevas habilidades mediante la creación de nuevos productos.

Los resultados también revelan que el uso de las redes sociales como herramienta para el cuidado y la gestión familiar da pie poco a poco al desarrollo de la identidad personal a través de la exploración de hobbies e intereses culturales. El siguiente extracto ilustra este proceso en Irene:

(I): (...) conecto mucho con una asociación por una enfermedad que tiene mi hija y me viene muy bien porque se ponen en contacto muchos enfermos. Sí, como un apoyo, me entienden, te diagnostican la enfermedad y te cae de sopetón y entonces pues te va explicando, porque tú sabes que los médicos y eso a lo mejor...pero necesitas más, ¿no? Y entonces pues ya te vas enterando de una, de otra, pues mira "yo tomo esto" "a mí me va bien esto, lo otro" vamos la enfermedad no es mía, es de mi hija, $¿$ ¿no? Y luego pues, con mucha gente a lo mejor por ejemplo también que yo pinto, nos mandamos los cuadros de una de otra, "pues yo estoy haciendo esto" "pues yo estoy haciendo éste otro" "pues yo he cogido ésta técnica de acrílico" "yo lo otro" (...)

Este extracto muestra cómo las redes sociales ha permitido a Irene gestionar mejor la salud de su hija a través de la participación activa en una asociación sobre su enfermedad, tomándola como fuente de información que le permite tomar

ICONO14 | Año 2015 Volumen 13 N² 2 | ISSN: 1697-8293 | DOI: ri14.v13i2.839 
decisiones más acertadas. Pero también muestra el sentido de realización personal que tiene para ella compartir sus intereses con otras personas.

Los relatos de las mujeres reflejan la sensación positiva que supone el uso de las redes sociales en la ampliación de su capital social, a través de las oportunidades que le ofrecen estas herramientas de aumentar su presencia y participación social más allá de su red íntima de contactos. Por un lado, estas mujeres revelan una mayor actividad y protagonismo en la vida social y cultural de sus comunidades locales, dinamizando y difundiendo a través de las redes iniciativas y prácticas culturales de sus comunidades. El extracto siguiente ilustra esta faceta.

(El): (...) el Facebook, hace mucho tiempo que lo utilizo porque en los años que aquí hubo de inmigración se fueron unas amigas mías muy jóvenes y las echaba mucho de menos, pero he sabido de ellas a través de terceras personas que venían. Total, que no sabíamos nada ninguna de nosotras y un día en el Facebook pues una de mis amigas que yo llevaba sin verla... pues veinticuatro años me reconoció, a partir de ese momento pues la verdad es que nos intercambiamos además de información muchas fotografías, porque ella lo que quiere es que le mande fotografías, fotografías de aquí del pueblo y mías porque yo participo en todas las cosas del pueblo. Si es carnaval, es carnaval, si es navidad, navidad lo que sea, y claro, como hay muchas fotografías, mucho material pues se lo mando y ella está encantada de la vida y, a través de eso ya llevamos nosotros un tiempo que nos comunicamos y sabemos de nuestras familias (...)

Por otro lado, este aumento de su capital social se vincula al sentido de realización personal que ellas le dan al poder participar activamente en grupos y comunidades con las que comparten hobbies e intereses comunes. Estos nuevos grupos les sirven como contexto de aprendizaje, crecimiento y empoderamiento personal. El siguiente fragmento muestra cómo su motivación por usar las redes sociales para desarrollar áreas de interés personal le lleva a una extensión de su capital social.

(A): Mis hijos no quieren que use Facebook, alguna cosa que dudo y que no sé cómo hacer, nada. No sé lo que se pensarán...querrán protegerme de que vaya a contactar conmigo alguien o yo que sé... Como soy presentadora de Tupperware

DOI: ri14.v13i2.839| ISSN: 1697-8293 | Año 2015 Volumen 13 N² 2 | ICONO14 
y Starhome, me llama mucha gente. El otro día me puso un "me gusta" una presentadora de Caracas...imagínate, pues ya me dijo mi hija, "mamá no te metas que verás...,ten cuidado...". Por supuesto, yo tengo cuidado...pero vamos no creo porque Facebook me ha servido para darme a conocer con mi trabajo y es muy útil. Antes trabajaba con Sevilla y ahora trabajo con Badajoz y en mi perfil de Facebook sobre Tupperware yo puedo subir fotografías de los productos que vendo, que a través de Internet busco de otras presentadoras en otros países y la gente por ahí hace cosas increíbles para vender, suben unas fotos preciosas de los productos adornados por Navidad y eso según la temporada o las promociones y yo eso... Y ahora estoy aprendiendo yo a hacer fotografía con el móvil y pasarla a mi Facebook para otras cosas que yo hago y que quiero que conozcan.

Por último, las redes sociales les posibilita la relación y el mantenimiento de grupos vinculados a su propia trayectoria vital (antiguos/as compañeros/as de la escuela, trabajo, etc.). Esta motivación por retomar contactos, es decir, recuperar el contacto con personas con las que ha compartido experiencias y vivencias en distintos ámbitos de actividad y etapas vitales supone también espacios para su participación e interacción social.

(D): Gracias a Internet he recuperado muchísima gente, tanto de mi primer matrimonio como del segundo y entonces...es muy bonito porque vuelves a ver gente que había desaparecido de tu vida. Estuve casada con un militar, eso te hace vivir en muchos sitios, compartir una serie de vivencias y tal, y eso me lo he vuelto a encontrar. (...) Entonces me encuentro con una serie de recuerdos y de emociones enormes que estoy feliz, y que comparto otra vez. (...) me han hecho volver atrás, muchos años. (...) Y ahora me vuelco, me llaman, me mandan, me dicen, "que hay aquí un concierto", allá que voy al concierto, también he jurado bandera hace dos años (...)

\section{Discusión y conclusiones}

A la luz de los resultados obtenidos en este estudio, podemos afirmar que las mujeres adultas de entorno rural muestran una variedad de motivos de uso de las redes sociales, destacando especialmente los motivos relacionales orientados a man-

ICONO14 | Año 2015 Volumen 13 Nº 2 | ISSN: 1697-8293 | DOI: ri14.v13i2.839 
tener y retomar contactos con familia y amigos. Esto concuerda con los resultados de investigaciones previas realizadas en estudiantes y adultos jóvenes (Arteaga et al., 2014; Hew \& Cheung, 2012; Lin \& Lu, 2011) y en mujeres (Jiménez-Cortés et al., 2015; Rebollo-Catalán \& Vico-Bosch, 2014) en las que se identifican motivos relacionales, de información y de entretenimiento en el uso de las redes sociales.

Los resultados de nuestro estudio indican que el fortalecimiento y consolidación de su red personal más íntima de familia y amigos constituye el entorno que propicia y motiva su acceso inicial a las redes sociales, mostrando además que parecen ser las redes intergeneracionales las más productivas para promover el acceso y uso de las redes sociales en mujeres adultas de entorno rural. Por otra parte, como las propias participantes afirman es el contacto con esta red más personal lo que les permite participar en la vida cultural y social de sus comunidades de origen a pesar de la distancia. El valor de las redes para traspasar los límites físico-geográficos y reforzar comunidades locales ayudando a mantenerlas unidas ha sido señalado con anterioridad (Castells, 2001; Herrero, Meneses, Valiente \& Rodríguez, 2004; Kollock \& Smith, 2003; Sloep \& Berlanga, 2011). En nuestro caso además, las mujeres de entorno rural destacan de forma muy especial la posibilidad de participar en las actividades y prácticas sociales y culturales de sus comunidades como una de sus principales motivaciones. En este sentido, también queremos señalar el papel que juegan las redes en el cuidado y gestión de la salud en la familia, reflejando estas mujeres en sus relatos el valor positivo que tiene en sus vidas participar en asociaciones y grupos en torno a la gestión y el cuidado de la salud. Algunos estudios previos (Godfrey \& Johnson, 2009) han documentado en personas mayores el efecto positivo de participar en comunidades virtuales a través de las redes sociales para el bienestar y el cuidado de su salud.

Los resultados de esta investigación también muestran el impacto positivo que supone en la vida de estas mujeres el uso de las redes sociales en la ampliación de su capital social a través de las oportunidades que le ofrecen estas herramientas de aumentar su presencia y participación social. Los relatos de estas mujeres reflejan cómo la entrada inicial en las redes motivada por su necesidad de estar en contacto con familiares y amigos da paso rápidamente a otras formas de uso. De esta forma, destaca el sentido de realización personal que tienen para ellas las redes sociales

DOI: ri14.v13i2.839| ISSN: 1697-8293 | Año 2015 Volumen 13 N² | ICONO14 
al poder compartir intereses, hobbies e inquietudes de índole cultural con otras personas, posibilitándoles esta interacción en las redes un refuerzo de su identidad personal. Estos resultados concuerdan con los de investigaciones previas (JiménezCortés et al., 2015; Joinson, 2008) que han demostrado la relación entre el uso de las redes y el refuerzo de la identidad mediante actividades que entrañan la participación en grupos con intereses afines. Los resultados de nuestro estudio indican que los hobbies e intereses (cocina, pintura, moda, viajes, etc.) son la vía de entrada para participar en estos grupos que les sirven como contexto de aprendizaje, crecimiento y empoderamiento personal. En esta vertiente, en los resultados de nuestro estudio resulta destacable cómo el acceso y uso de estas mujeres a las redes sociales las vuelve más activas y creativas en diferentes ámbitos de la vida cultural, social y laboral en sus comunidades. Dos aspectos sobresalen de los relatos de las mujeres rurales entrevistadas para este estudio: por una parte, su compromiso e implicación en las iniciativas y proyectos culturales de sus comunidades con prácticas de dinamización y difusión que realizan a través de las redes y, por otra, el uso de las redes para mejorar su actividad a nivel local a través de la interacción con comunidades de otros contextos lejanos geográficamente que les proporcionan ideas, recursos, etc. que puede emplear y poner a disposición de su comunidad. Esta idea del rol activo de las personas que transforman y producen conocimiento mediante su participación e interacción en las redes sociales ha sido planteada con anterioridad como algo que es característico de la naturaleza y dinámica que plantean los entornos mediados con estas tecnologías digitales interactivas (Aires et al., 2014; Meyers et al., 2013; Simsek \& Simsek, 2013).

A partir de los resultados de este trabajo podemos concluir que las mujeres adultas de zonas rurales inician el uso de las redes sociales por la necesidad de responder a propuestas y demandas de su entorno familiar y afectivo más íntimo, aunque rápidamente descubren nuevas posibilidades en contacto con esta herramienta, posibilitándoles cubrir diversos planos que van desde la información y el aprendizaje, la participación cultural y social y el desarrollo de la propia identidad a través de intereses y hobbies, siendo precisamente estas nuevas funciones las que propician un aumento de su capital social. Los estudios sobre alfabetización digital y nuevas alfabetizaciones (Meyers et al., 2013; Simsek \& Simsek, 2013; Area \& Pessoa, 2012; Sloep \& Berlanga, 2011) insisten en la importancia de contemplar, más allá que la pura y exclusiva adquisición

ICONO14 | Año 2015 Volumen 13 N² | ISSN: 1697-8293 | DOI: ri14.v13i2.839 
de competencias, la participación en culturas y prácticas digitales como parte esencial de la educación que pretenda una ciudadanía digital plena, activa y responsable.

\section{Referencias}

Aires, L., Dias, P., Azevedo, J., Rebollo-Catalán, A. \& García-Pérez, R. (2014). Education, Digital Inclusion and Sustainable Online Communities. En S. Caeiro, W. Leal Filho, U. M. Azeiteiro, (Eds.), E-learning and sustainability ", series Umweltbildung, Umweltkommunikation und Nachhaltigkeit - Environmental Education, Communication and Sustainability. New York: Peter Lang Publishers. Area, M. \& Pessoa, T. (2012). De lo sólido a lo líquido: las nuevas alfabetizaciones ante los cambios culturales de la Web 2.0. Comunicar, 38, 13-20. doi: 10.3916/ C38-2012-02-01

Arteaga, R., Cortijo, V. \& Javed, U. (2014). Students' perceptions of Facebook for academic purposes. Computers \& Education, 70, 138-149. doi: 10.1016/j. compedu.2013.08.012

Baker, P., Bricout, J., Moon, N., Coughlan, B. \& Pater, J. (2013). Communities of participation: A comparison of disability and aging identified groups on Facebook and LinkedIn. Telematics and Informatics, 30(1), 22-34. doi: 10.1016/j.tele.2012.03.004

Bimber, B. (2000). Measuring the Gender Gap on the Internet. Social Science Quarterly, 81(3), 868-876.

Bradley, N., \& Poppen, W. (2003). Assistive technology, computers and internet may decrease sense of isolation for homebound elderly and disabled persons. Technology and Disability, 15(1), 19-25.

Braun, M. T. (2013). Obstacles to social networking website use among older adults. Computers in Human Behavior, 29(3), 673-680. doi: 10.1016/j.chb.2012.12.004

Carpenter, B. D. \& Buday, S. (2007). Computer Use among Older Adults in a Naturally 0ccurring Retirement Community. Computers in Human Behavior, 23(6), 30123024. doi: 10.1016/j.chb.2006.08.015

Castells, M. (2001). ¿Comunidades virtuales o sociedad red? En M. Castells, La Galaxia Internet: Reflexiones sobre Internet, empresa y sociedad. Madrid: Areté.

Dias, I. (2012). 0 uso das tecnologías digitais entre os seniores. Motivaçoes e intereses. 
Sociología, problemas e práticas, 68, 51-77. doi: 10.7458/SPP201268693 DiMaggio, P., Hargittai, E., Celeste, C. \& Shafer, S. (2004). Digital Inequality: From Unequal Access to Differentiated Use. In K. Neckerman (Ed.), Social Inequality, (pp. 355-400). New York: Russell Sage Foundation.

Falk, I. \& Kilpatrick, S. (2000). What is social capital? A study of interaction in a rural community. Sociologia Ruralis, 40(1), 87-110.

García, A., López de Ayala, M.C. \& Catalina, B. (2013). Hábitos de uso en Internet y en las redes sociales de adolescentes españoles. Comunicar, 41, 195-204. doi: 10.3916/C41-2013-19

Godfrey, M. \& Johnson, 0. (2009). Digital circles of support: Meeting the information needs of older people. Computers in Human Behavior, 25(3), 633-642. doi: 10.1016/j.chb.2008.08.016

Greenhow, C. \& Gleason, B. (2012). Twitteracy: Tweeting as a New Literacy Practice. The Educational Forum, 76(4), 464-478. doi: 10.1080/00131725.2012.709032 Greenhow, C. \& Burton, L. (2011). Help from my "friends": Social capital in the social network sites of low-income students. Journal of Educational Computing Research, 45(2), 223-245. doi: 10.2190/EC.45.2.f

Greenhow, C. \& Robelia, B. (2009). Informal learning and identity formation in online social networks. Learning, Media and Technology, 34(2), 119-140. doi: $10.1080 / 17439880902923580$

Hall, R. (2009). Towards a Fusion of Formal and Informal Learning Environments: the Impact of the Read/Write Web. Electronic Journal of e-Learning, 7(1), 29-40. Hargittai, E. (2010). Digital Na(t)ives? Variation in Internet Skills and Uses among Members of the «Net Generation». Sociological Inquiry, 80(1), 92-113. doi: 10.1111/j.1475-682X.2009.00317.x

Herrero, J., Meneses, J., Valente, L. \& Rodríguez, F. (2004). Participación social en entornos virtuales. Psicothema, 16(3), 456-460.

Hew, K. F. \& Cheung, W. S. (2012). Use of Facebook: a case study of Singapore students' experience. Asia Pacific Journal of Education, 32(2), 181-196. doi: 10.1080/02188791.2012.685560

Hilbert, M. (2011). Digital gender divide or technologically empowered women in developing countries? A typical case of lies, damned lies, and statistics. Women's Studies International Forum, 34(6), 479-489. doi: 10.1016/j.wsif.2011.07.001

Huber, L. \& Watson, C. (2014). Technology: Education and Training 
Needs of Older Adults. Educational Gerontology, 40(1), 16-25. doi: 10.1080/03601277.2013.768064

Jiménez-Cortés, R., Rebollo-Catalán, A., García-Pérez, R. \& Buzón-García, 0. (2015).

Motivos de uso de las redes sociales virtuales: Análisis de perfiles de mujeres rurales. RELIEVE, 21(1). doi: 10.7203/relieve.21.1.5153

Joinson, N. A. (2008). Looking at', 'Looking up' or 'Keeping up with' People? Motives and uses of Facebook. CHI 2008 Proceedings: 1027-1036.

Kilpatrick, S., Field, J. \& Falk, I. (2003). Social Capital: an analytical tool for exploring lifelong learning and community development. British Educational Research Journal, 29(3), 417-433. doi: 10.1080/0141192031000156024

Kollock, P. \& Smith, M.A. (2003). Comunidades en el ciberespacio. Barcelona: UOC. LaRose, R., Gregg, J. L., Strover, S., Straubhaar, J. \& Carpenter, S. (2007). Closing the rural broadband gap: Promoting adoption of the Internet in rural America. Telecommunications Policy, 31(6-7), 359-373. doi: 10.1016/j.telpol.2007.04.004 Lin, K. \& Lu, H. (2011). Why people use social networking sites: An empirical study integrating network externalities and motivation theory. Computers in Human Behavior, 27(3), 1152-1161. doi: 10.1016/j.chb.2010.12.009

Livingstone, S. \& Helsper, E. (2007). Gradations in digital inclusion: Children, young people, and the digital divide. New Media and Society, 9(4), 671-696. doi: $10.1177 / 1461444807080335$

Mayorga, M. J. (2004). La entrevista cualitativa como técnica de la evaluación de la docencia universitaria. RELIEVE, 10(1), 23-39.

Meyers, E. M., Erickson, I. \& Small, R. V. (2013). Digital literacy and informal learning environments: an introduction. Learning, Media and Technology, 38(4), 355-367. doi: 10.1080/17439884.2013.783597

Muscanell, N. \& Guadagno, R. (2012). Make new friends or keep the old: Gender and personality differences in social networking use. Computers in Human Behavior, 28(1), 107-112. doi: 10.1016/j.chb.2011.08.016

Novo-Corti, I., Varela-Candamio, L. \& García-Álvarez, T. (2014). Breaking the walls of social exclusion of women rural by means of ICTs: The case of "digital divides" in Galician. Computers in Human Behavior, 30, 497-507. doi: 10.1016/j. chb.2013.06.017

Papacharissi, Z. \& Mendelson, A. (2011). Toward a new(er) sociability: uses, gratifications and social capital on Facebook. In S. Papathanassopoulos (Ed.), 
161 | Luisa Vega Caro, Alba Vico Bosch y Ángeles Rebollo Catalán

Media Perspectives for the 21st Century (pp. 212-230). New York: Routledge. Pfeil, U., Arjan, R. \& Zaphiris, P. (2009). Age differences in online social networking - A study of user profiles and the social capital divide among teenagers and older users in MySpace. Computers in Human Behavior, 25(3), 643-654. doi: 10.1016/j.chb.2008.08.015

Plan de inclusión digital y empleabilidad (2013). Ministerio de Industria, Energía y Turismo. Recuperado de http://www.agendadigital.gob.es/planesactuaciones/ Bibliotecainclusion/1.\%20Plan/Plan-ADpE-7_Inclusion-Empleabilidad.pdf

Rebollo-Catalán, A. \& Vico-Bosch, A. (2014). El apoyo social percibido como factor de la inclusión digital de las mujeres de entorno rural en las redes sociales virtuales. Comunicar, 43, 173-180. doi: 10.3916/C43-2014-17

Sánchez-Franco, M. J., Buitrago-Esquinas, E. M. \& Hernández-Mogollón, J. M. (2012). Antecedentes sociales y psicológicos del compromiso comunitario. Un análisis del comportamiento del usuario de una red social de relaciones. Cuadernos de Economía y Dirección de la Empresa, 15, 205-220. doi: 10.1016/j. cede.2012.04.007

Sancho, J. M. (2009). Dones a la ciència i la universitat: la construcció de la identitat docent $i$ investigadora en contextos de desigualtat. Barcelona: AGAUR.

Simsek, E. \& Simsek, A. (2013). New Literacies for Digital Citizenship. Contemporary Educational Technology, 4(2), 126-137.

Sloep, P. \& Berlanga, A. (2011). Redes de aprendizaje, aprendizaje en red. Comunicar, 37, 55-64. doi: 10.3916/C37-2011-02-05

Straubhaar, J., Spence, J., Tufekci, Z. \& Lentz, R. (Eds.) (2012). Inequity in the technopolis. Race, Class, Gender, and the Digital Divide in Austin. Austin: University of Texas Press.

Strauss, A. \& Corbin, J. (1998). Basics of qualitative research. Techniques and procedures for developing grounded theory. London: Sage.

Tosun, L. P. (2012). Motives for Facebook use and expressing "true self" on the Internet. Computers in Human Behavior, 28(4), 1510-1517. doi: 10.1016/j. chb.2012.03.018

van Dijk, J. (2005). The deepening divide: inequality in the information society. Thousand Oaks, CA: Sage Pub.

Vázquez-Gómez, G. (2003). Sociedad-red, ciudadanía cognitiva y educación. Revista de Educación, núm. extraordinario, 13-31.

ICONO14 | Año 2015 Volumen 13 Nº 2 | ISSN: 1697-8293 | DOI: ri14.v13i2.839 
Vergés, N., Hache, A. \& Cruells, E. (2011). Indagando en la relevancia de Internet en el acceso, uso y deseos de las TIC por parte de las mujeres en las TIC. Teoría de la Educación: Educación y Cultura en la Sociedad de la Información, 12(2), 105-121.

Wagner, I., Birbaumer, A. \& Tolar, M. (2003). Widening Women's Work in Information and Communication Technology. Professional trajectories and Biographies. Information Society Technologies Programme (IST Fifth Framework Programme, European Commission, DG Information Society).

Yang, C. C \& Brown, B. (2013). Motives for Using Facebook, Patterns of Facebook Activities, and Late Adolescents. Social Adjustment to College. Journal of Youth Adolescence, 42, 403-416. doi: 10.1007/s10964-012-9836-x

\section{Agradecimientos}

Este trabajo se ha podido realizar gracias al Proyecto de Investigación de Excelencia titulado «Calidad relacional, inmersión digital y bienestar social desde una perspectiva de género. Una aplicación de las redes sociales on-line en la mujer rural andaluza», financiado en la convocatoria de Proyectos de Excelencia 2011-15 de la Junta de Andalucía (España) (P10-SEJ-5801) y al Proyecto de I+D «Las mujeres como tejedoras de las redes sociales: estrategias relacionales e inclusión digital», financiado en la convocatoria de Proyectos I+D 2013 del Ministerio de Economía y Competitividad (España) (EDU2013-45134-P). Este trabajo se basa parcialmente en el trabajo de tesis doctoral de Alba Vico Bosch, que realiza con un contrato predoctoral del Programa FPU, bajo la dirección de Ángeles Rebollo Catalán.

DOI: ri14.v13i2.839| ISSN: 1697-8293 | Año 2015 Volumen 13 N² 2 | ICONO14 